\title{
SISM: Software e Medidor Inteligente para Acompanhamento do Consumo Residencial de Energia Elétrica
}

\author{
Rafael Cavalheiro, Filipe Saraiva \\ Faculdade de Computação - Instituto de Ciências Exatas e Naturais \\ Universidade Federal do Pará (UFPA) \\ Belém - Pará - Brasil \\ rafcavalheiro@gmail.com, saraiva@ufpa.br
}

\begin{abstract}
This article presents a web system integrated to a prototype electric energy meter for the monitoring of consumption in household appliances. Measurement data is collected and sent by communication network to the system, which provides the user with real-time information on consumption and charging, assisting him in making decisions about the use of electric power in his residence. Thus, this tool intends to assist the user for the conscious and sustainable use of electric energy.
\end{abstract}

Resumo. Este artigo apresenta um sistema web integrado a um protótipo de medidor de energia elétrica para o monitoramento do consumo em eletrodomésticos. Os dados de medição são auferidos e enviados via rede de comunicação para o sistema, que apresenta ao usuário informações em tempo real sobre consumo e tarifação, auxiliando-o na tomada de decisão sobre o uso de energia elétrica em sua residência. Assim, essa ferramenta pretende assistir o usuário para o uso consciente e sustentável de energia elétrica.

\section{Introdução}

O desenvolvimento sustentável nas metrópoles requer mudanças que necessitam de planejamento, investimentos e soluções eficientes que viabilizem estratégias para estruturar áreas como espaço urbano, meio ambiente, energia, telecomunicações e outras. Essas mudanças são obtidas a partir de inovações em processos e tecnologias, que devem ter o meio ambiente e a sustentabilidade como foco.

Em termos do setor elétrico, os sistemas de informação estão se tornando protagonistas por permitir o gerenciamento da rede e prover novas funcionalidades. Os chamados smart grids são redes de energia elétrica onde existem dispositivos capazes de realizar medições e troca de dados entre si [Schettino 2014]. Essa possibilidade permite o desenvolvimento de diversas aplicações, tanto em nível da rede em si, como técnicas de autorrecuperação após faltas na distribuição de energia [Campos and Saraiva 2018], quanto em nível de usuário, como na análise e gerenciamento da demanda residencial pelo lado do consumidor [Pipattanasomporn et al. 2012].

Com o aumento do consumo de energia elétrica, são necessárias as construções de novas usinas e, com elas, sempre há consequências e impactos para o meio ambiente [Pinheiro and Kohlrausch 2011]. Mesmo as hidrelétricas, majoritárias na matriz elétrica nacional, demandam grandes barragens que afetam populações e a natureza. Com o objetivo de difundir estratégias e ferramentas para auxiliar o consumo mais sustentável e 
consciente, este artigo apresenta o desenvolvimento de um protótipo de medidor de energia e um sistema de informação próprios para assistir a essa tarefa.

O medidor produzido nesta pesquisa é batizado de Electabuzz e foi construído em plataforma Arduino [McRoberts 2015]. O dispositivo funciona como um sensor junto ao Sistema de Informação Integrado a Smart Meter (SISM), um sistema web que tem a finalidade de processar os dados coletados pelo Electabuzz de forma a apresentar ao usuário o consumo elétrico de eletrodomésticos e a tarifa em tempo real, possibilitando tomadas de decisão sobre o consumo de maneira mais sustentável e estratégica.

Nas seções seguintes serão descritos o protótipo do Electabuzz (Seção 2) e as funcionalidades do SISM (Seção 3). Ao final, serão discutidas conclusões e trabalhos futuros.

\section{Electabuzz: um protótipo de medidor de energia elétrica}

O Electabuzz é um protótipo de medidor de energia elétrica que pode ser considerado "inteligente" (smart meter) devido a sua capacidade de comunicação de dados via rede [Zheng et al. 2013]. Ele foi desenvolvido utilizando Arduino pois essa plataforma facilita a conexão com diversos dispositivos e ferramentas de software, além de ter baixo custo.

Os principais componentes usados na construção do protótipo são: Placa Arduino Uno R3, Ethernet Shield W5100, Sensor de Corrente Não Invasivo SCT-013 20A, Display LCD 16x2 Backlight Azul, Barra de Pinos 1x40 180 Graus, Protoboard 830 pontos, Potenciômetro Trimpot $10 \mathrm{~K}$, resistor $10 \mathrm{~K} 1 / 4 \mathrm{~W}$, além de componentes auxiliares como conectores elétricos, USB, fontes de energia e cabo tipo par trançado.

Durante a operação, o Electabuzz registra consumos de energia de determinado equipamento e envia essas medições para um banco de dados, que depois será utilizado pelo SISM. A Figura 1 apresenta as conexões lógicas realizadas para a construção do Electabuzz.

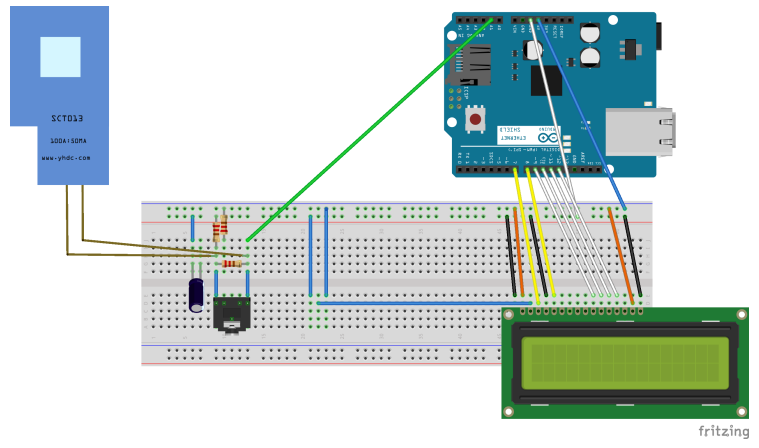

Figura 1. Conexão lógica do Electabuzz

\section{SISM: Sistema de Informação Integrado a Smart Meter}

SISM é um sistema web escrito em PHP, responsivo, que pode ser acessado por qualquer navegador de computadores ou dispositivos móveis. O SISM opera sobre o banco de dados com as medições elétricas alimentadas pelo Electabuzz.

No SISM, o usuário deve primeiramente através da interface web cadastrar o equipamento que será monitorado. Os equipamentos cadastrados podem ser de diversos tipos 
e classes energéticas: geladeiras, televisores, ventiladores, entre outros. Para cada equipamento cadastrado é gerado um código único que deve ser informado ao Electabuzz.

Feito isso, a tomada de energia convencional do equipamento deve ser plugada ao Electabuzz para que ele possa enviar dados de medição pela rede ao banco de dados. A partir desses monitoramentos, o SISM gera gráficos de consumo e realiza cálculos de tarifa em tempo real. O SISM é capaz de realizar medições simultâneas para vários equipamentos, requerendo apenas a disponibilidade de medidores Electabuzz.

A Figura 2 apresenta o sistema como um todo funcionando de maneira integrada.

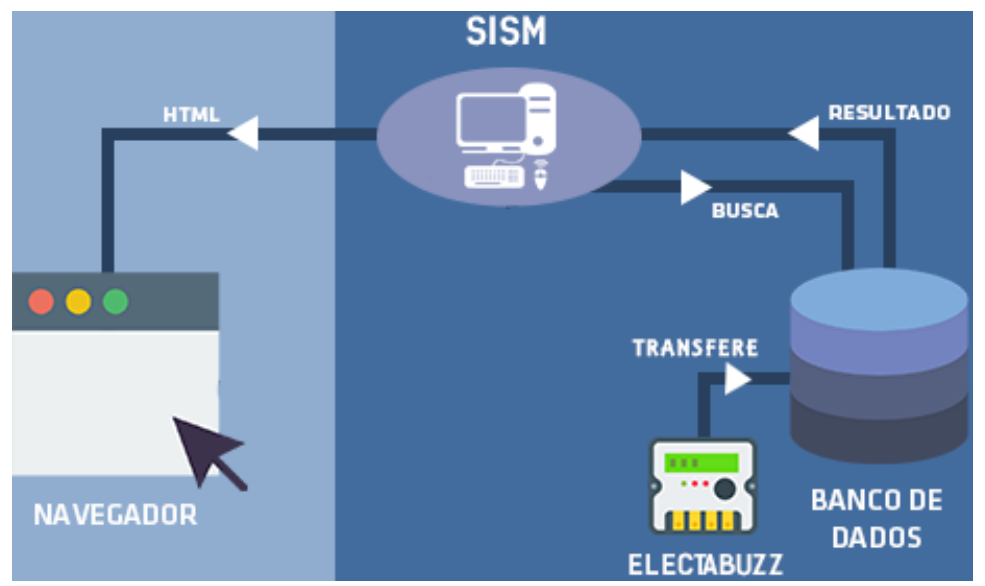

Figura 2. Integrações do SISM e Electabuzz

A Figura 3 apresenta algumas telas do SISM acessado via celular. Figura 3A é a tela de login, Figura 3B a área do usuário, Figura 3C é a tela de cadastro de equipamento, e finalmente a Figura 3D é a tela de monitoramento.
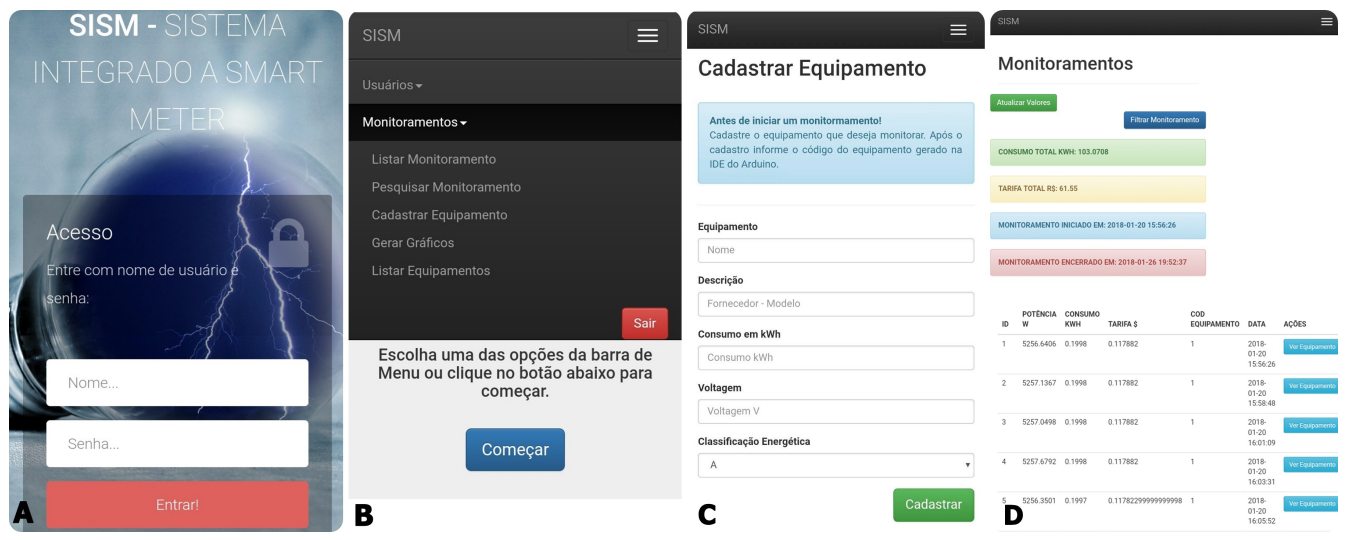

Figura 3. Tela de Login do SISM (A), Área do usuário (B), Cadastro de Equipamento (C), Tela de monitoramento (D)

A Figura 4 exemplifica os consumos e tarifas de cada utilização obtidas a partir do monitoramento de uma máquina de lavar roupa.

Além dos gráficos e valores por cada utilização, o SISM também apresenta o custo total do consumo dos equipamentos monitorados na tarifa para dado mês e um gráfico 


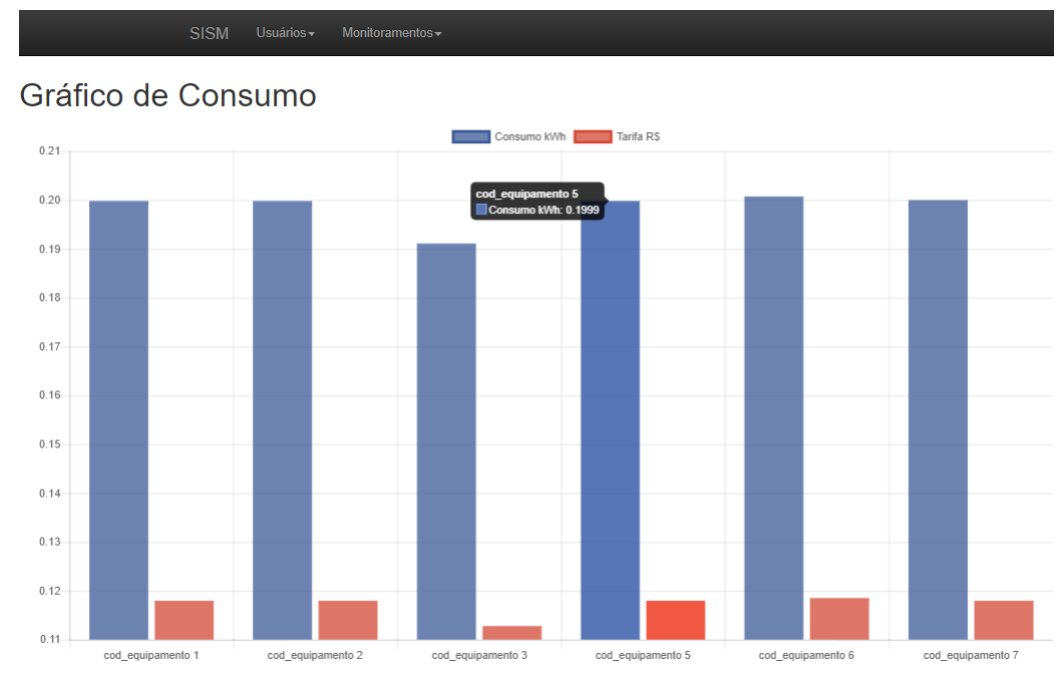

Figura 4. Tela de monitoramento para uma máquina de lavar

comparativo do consumo entre estes equipamentos. O SISM também realiza cadastros, alterações e remoções de usuários e gerenciamento de senhas para acesso.

\section{Conclusão}

O SISM é um sistema web que, combinado com o medidor Electabuzz, é capaz de apresentar ao usuário medições de consumo e tarifação em tempo real de eletrodomésticos. Essa ferramenta objetiva prover ao usuário informações suficientes que o subsidiem para tomada de decisão sobre o consumo de energia elétrica em sua residência, possibilitando o uso consciente e sustentável da mesma.

Como trabalhos futuros, objetiva-se criar grandes bases de dados sobre consumo residencial utilizando o SISM e Electabuzz, e também adicionar funcionalidades ao SISM para criação de estratégias sustentáveis de consumo utilizando métodos de inteligência computacional como lógica fuzzy.

\section{Referências}

Campos, I. R. and Saraiva, F. (2018). Proposta de modelo de autorrecuperação de sistemas de distribuição de energia elétrica utilizando sistemas multiagente. Workshop de Sistemas de Agentes, seus Ambientes e aplicações - WESAAC, pages 244-249.

McRoberts, M. (2015). Arduino Básico. Novatec.

Pinheiro, D. K. and Kohlrausch, F. (2011). Educação ambiental: Uso consciente da energia elétrica e aplicação de alternativas para diminuição de consumo. Revista Eletrônica em Gestão, Educação e Tecnologia Ambiental, 4(4):387-397.

Pipattanasomporn, M., Kuzlu, M., and Rahman, S. (2012). An algorithm for intelligent home energy management and demand response analysis. IEEE Transactions on Smart Grid, 3(4):2166-2173.

Schettino, S. (2014). Smart Grid: tendências de sua implantação no Brasil. Appris.

Zheng, J. et al. (2013). Smart meters in smart grid: An overview. IEEE Green Technologies Conference, pages 57-64. 